\title{
Modelo de comportamiento de las bibliotecas públicas cubanas y su indice representativo
}

\section{Emilio Setién Quesada}

Investigador Titularde la Biblio teca Na cio nal José Mar tí. Pla za de la Revolución, La Habana, Cuba.

e-mail:setien@binanet.cu

\section{RESUMEN}

$\boldsymbol{S}$ e presen ta el re sul ta do prin ci pal de las in ves ti ga cio nes so bre mo de la ción ma temáti ca de las bi blio te cas pú bli cas cu ba nas en sus con di cio nes ac tua les de de sa rro1lo, una vez perfec cio na do des pués de su uti li za ción en la prác ti ca por cer ca de 5 años. El mo de lo in clu ye va ria bles que re pre sen tan los ele men tos esen cia les de la actividad bibliotecaria y reproduce las relaciones que se establecen entre ellos. Pue de ser apli ca do to man do como base tres ti pos de va lo res: los que ri gen el de sarrollo perspectivo de las bi blio te cas; los que res pon den a pe rio dos; y los que responden a biblio te cas dis tin tas. Se in clu ye un ejem plo de apli cación.

\section{ABSTRACT}

Improved af ter aproxi ma tely five years of prac ti cal test, the main issue of re search on mathe ma tical mo dels for the $\mathrm{Cu}$ ban public libraries, in their pre sent de ve lo ping conditions, is described. The mo del in clu des va riables which re pre sent the esential elements of library activity and reproduces the relations among them. It may be applied using three diffe rent ba sis: va lues es ta blished for the fu tu re de ve lopment of libraries; values for the beha vior in diffe rent pe riods and va lues in diffe rent libraries. An application sample is in cluded.

$\mathrm{L}$ as investigaciones que dieron origen $\checkmark$ al re sul ta do que nos ocu pa se efec tuaron entre 1985 y 1989, como parte del programa científico de la Dirección de Bibliotecas del Ministerio de Cultura de Cuba, que es tu vo in te gra do por las ta reas siguientes:

el es tu dio de los ras gos esen cia les de la actividad bibliotecaria y de otras formas de comunicación social que le son con ti guas, asícomo del con te ni do, carácter y estructura de la Bibliotecología; ${ }^{1}$
- la caracterización del estado actual de la base de datos estadísticos sobre las biblio te cas públicas cubanas y la forma en que éstos permiten reflejar el nivel de de sarrollo de esas ins ti tu ciones, con el fin de definir las posibilidades de aplicación de modelos matemáticos que se apoyan en con cep tos ase quibles a la medición, y para determinar la dirección a la que debe orientarse esa aplicación $;^{2}$

- el aná li sis críti co de los mo de los localizados en la literatura consultada sobre el tema, que permitenevaluarsituaciones concretas de la actividad bibliotecaria y que, por ofrecer un índice integral de diver sos ele men tos y re la ciones esenciales de esa actividad, sirvenpara comparar el comportamiento de unas bibliotecas y otras;

el diseño del modelo de comportamiento de las bibliotecas públicas cubanas y su índice representativo, producto principal de esas investigaciones, que ahorasepresenta.

1 Emilio Setién. Práctica bibliotecaria y teoría bibliotecológica. - Ciudad de La Habana : Universidad de La Habana, 1989. — 21p. Los resultados obtenidos que se reflejan en esta obra han sido perfeccionados y se encuentran en vías de publicación por la Biblioteca Nacional José Martí. No obstante, su consulta es útil para conocer mejor el sustento teórico del modelo matemático del comportamiento de las bibliotecas públicas cubanas

2 _ _ . "Estado de desarrollo de las bibliotecas públicas cubanas: condiciones para la modelación matemática de su actividad." En: Investigación Bibliotecológica (México, D.F.) 5(11):24-32;jul.-dic., 1991. 


\begin{tabular}{|c|c|c|}
\hline \multicolumn{3}{|c|}{ Tabla 1} \\
\hline \multicolumn{3}{|c|}{ Eficiencia } \\
\hline & Esfuerzos & Efectividad \\
\hline Elementos & $\begin{array}{l}\text { Colecciones }(f) \\
\text { Bibliotecarios }(B)\end{array}$ & $\begin{array}{l}\text { Préstamos }(m) \\
\text { Lec tores potenciales }(N) \\
\text { Lec to res rea les }(n) \\
\text { Lec to res que uti li zan el fon do }(l)\end{array}$ \\
\hline Relaciones & $\begin{array}{c}\text { Rota ción de colec ciones }(R=m / f) \\
\text { Productividad }(f / B, m / B, N / B, \\
n / B, l / B)\end{array}$ & $\begin{array}{l}\text { Cir cu la ción }(\mathrm{C}=\mathrm{m} / \mathrm{l}) \\
\text { Comunicación }(\alpha=l / n) \\
\text { Captación }(\alpha,=n / N)\end{array}$ \\
\hline
\end{tabular}

El modelo matemático de comportamiento de las bibliotecas públicas cubanas está des ti na do a ofre cer un ín di ce integral del comportamiento de la eficiencia real de esas instituciones en com pa ra ción con va lo res de base de distinta índole.

Según Wersig y Neveling, eficiencia es la relación entre los esfuerzos del sistemay su efec ti vi dad. ${ }^{3}$ A esta de fi ni ción se ajusta el diseño del modelo.

Los esfuerzos son los mediosempleados por las bibliotecas para cumplir su misión, expresados fundamentalmente en me dios ore cur sos de colec ción $(f)$, re cursos de personal $(B)$ y en su utilización o rendimiento.

De acuerdo con la práctica de otros países y con la experiencia cubana, los recursos de las bibliotecas se destinan en un 70 a un $90 \%$ al per so nal y a la ad qui sición de fon dos, por lo que los re cur sos de colección $(f)$ y de personal $(B)$ son los medios de más peso en la actividad bibliotecaria. ${ }^{4}$

La efecti vi dad de las bi blio te cas se sin teti za en los prés ta mos $(m)$ y se re fle ja, asimismo, en la cantidad de lectores $(l)$ que se benefician de ellos, en la medida en que los lectores inscritos en las bibliotecas $(n)$ se in cor po ran al sis te ma de co municación social autor-lector que propician esas instituciones ( $\alpha=l / n)$ y en el poder de captación de los lectorespotenciales $(N)$ de éstas $(\alpha,=n / N)$.

Los ele men tos y re la cio nes esen cia les de la actividad bibliotecaria, que expresan con cep tos ase qui bles a la me di ción, pueden agru par se, en tér mi nos de efi cien cia, como se expresa en la tabla 1 .
En esta tabla quedan expresados los criterios de eficiencia de las bibliotecas, porque:

El índice de rotación expresa los es fuerzos fundamentales del sistema, refle ja dos en el ni velde sus co lec ciones y en la frecuencia de uso de éstas.

El índice de circulación refleja el impacto de estos esfuerzos en el público $\mathrm{y}$, consecuentemente, el resultado del trabajo de promoción de las bibliotecas.

La efec ti vi dad del tra ba jo de las bi bliotecas, que se sintetiza en el índice de circulación, queda reflejada, además, en dos momentos previos al acto de préstamo: en el nivel alcanzado por la orientación al lector, expresado en el índice de comunicación, y en el nivel al can za do en la in cor po ra ción de la comunidad a la biblioteca, cuya medida está dada por el índice de captación.

Como no escapa a la comprensión del lec tor es pecializado, es tosín dices sonlos más representativos del trabajo biblioteca rio, en esen cia, y son, al mis mo tiem po, componentes del concepto de eficiencia adoptado en la investigación.

Nótese que la simbología $l, n$ y $N$ ha sido utilizada para identificar distintas catego rías de lec tores: lec to res que uti li zan el fondo $(l)$, lectores reales inscritos en las bibliotecas $(n)$, lectores potenciales $(N)$. Las dos últimas ( $n$ y $N$ ) son empleadas tam bién por otros au to res. Pero mien tras que para el modelo de comportamiento de las bibliotecas públicas cu ba nas el índice de circulación se calcula a partir de los lectores que utilizan el fondo $(C=$ $m / l$ ), porque se identifica así la frecuencia real de lec tu ra por usua rio, otros au tores cal cu lan ese ín di ce res pec to a los lec- tores inscritos (hagan uso de las colecciones o no) $(C=m / n)$, y otros más lo ha cen res pec to a los lec to res po ten ciales $(C=m / N)$. Estas dos últimas formas de calcu lar elín di ce de cir cu la ción no satisfacen las necesidades de información de las bibliotecas públicas cubanas, porque sólo reflejan frecuencias de lectura es ti ma das y no rea les. El em pleo de $N$ y $n$ en el cál cu lo delín di ce de cir cu la ción se podrá apreciar más adelante, durante el aná li sis que se ofre ce de mo de los que sirvieron de antecedentes al actual.

A los efectos del modelo de comportamiento de las bibliotecas públicas cubanas, los valores totales de los préstamos $(m)$, las colecciones $(f)$, los lectores que utilizan el fondo $(l)$, los lectores reales ins cri tos en las biblio te cas $(n)$ y los lec tores potenciales $(N)$ se sustituyen por los promedios de esos valores por bibliotecario, con el fin de expresar la productivi dad de és tos como una de las re la cio nes básicas correspondientes a los esfuerzos del con cep to de efi cien cia adop ta do. Este tratamiento constituye una innovación res pec to a la re presen ta ción uti li za da por otros autores para las variables antes mencionadas, cuando las utilizan.

El he cho de que en el mo de lo de com portamiento se empleen promedios por biblio te cario, en lu gar de los valores to tales correspondientes a cada variable, como lo ha cen esos au to res, no al te ra el re sul tado que se ob tie ne para los ín di ces de ro tación $(R)$, circulación $(C)$, comunicación $(\alpha)$, cap ta ción $(\alpha$,$) , lo que se pue de com-$ probar fácilmente. Nótese, por ejemplo que:

$$
R=\frac{\bar{m}}{\bar{f}}=\frac{\frac{m}{B}}{\frac{f}{B}}=\frac{m \cdot B}{B \cdot f}=\frac{m}{f}
$$

Las operaciones mate máticas que se pueden efectuar con índices calculados sobre la base de promedios y con aquellos que se apoyan en cifras absolutas, así como las conclusiones a que se llega a partir de los resultados obtenidos con esas operaciones son, entonces, equivalentes. Esa equivalencia permite sustituir, llegado el caso, a cada una de esas expresiones por la otra.

3 Véase Wersig, G. y U. Neveling. Terminology of Documentation. — París : The Unesco Press, 1976. — p. 167-168.

4 Cuba. Ministerio de Cultura. Dirección de Bibliotecas. Circular sobre el presupuesto de las bibliotecas públicas. - Ciudad de La Habana, 1984. - $2 \mathrm{~h}$ 


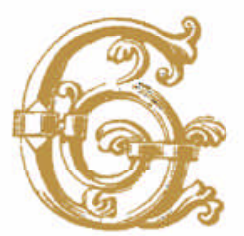

Se considera como lectores potenciales a los habitantes que residen en el radio de acción de 2 km como máximo, alrededor de cada biblioteca pública, para los que se cuenta con fondo suficiente

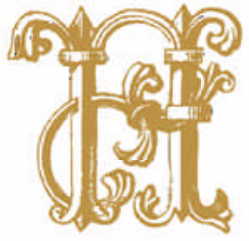

La innovación antes descrita es útil porque, al ofrecer información so bre el rendi mien to de los bi blio te ca rios res pec to a las variables que representan elementos esenciales de la actividad bibliotecaria, se refleja el papel que desempeña ese profesional como elemento activo que in ter vie ne en el de sa rro llo de las re la ciones fundamentales que caracterizan esa actividad (lector-préstamo-bibliotecario-colecciones), aunque la cantidad de ellos no apa rez ca di rec ta men te en el modelo.

El modelo de comportamiento de las bibliotecas públicascubanasestáformado, por tan to, por las va ria bles que re pre sentan elementos esenciales de la actividad bibliotecaria, en términos de promedios por bibliotecario; y son:

$\bar{m}=$ prome dio de prés ta mos por biblio tecario;

$\bar{f}=$ volú me nes en el fon do por bi blio tecario;

$\bar{l}=$ promedio de lectores que utilizan el fondo por bibliotecario;

$\bar{n}=$ promedio de lectoresrealesinscritos por bibliotecario;

$\bar{N}=$ promedio de lectores potenciales por bibliotecario.

Estos símbolos, cuando están acompaña dos porel subín dicei $\left(\bar{m}_{i}, \bar{f}_{i}\right.$, etcétera), indican valores que se toman como base para comparar los valores reales de las variables que integran el modelo en un momento dado. Esos valores pueden ser de índole diversa:

- Valores que se toman en cuenta en las disposiciones que rigen el desarrollo perspecti vode las bibliote cas públicas, o los que se pueden derivar de ellas.

- Valores de esas va riables en pe rio doso bibliotecas distintos al caso que se estudia.

Elín di ce de com por ta mien to es una función de variables presentes en la actividad bibliotecaria que expresan su eficiencia. Es tas variablesinte gran tam bién índices de aspectos parciales:

El índice de rotación:

$$
\frac{R}{R_{i}}=\frac{\frac{\bar{m}}{\overline{\bar{f}}}}{\frac{\bar{m}_{i}}{\bar{f}_{i}}}
$$

cuya expresión ofre ce la me di da com pa rada de la intensidad con que se utilizan las colecciones en dos momentos distintos (frecuencia de préstamos por volumen), in di can do al mis mo tiem po el rendi mien to por bi blio te ca rio en cada una de las variables utilizadas; el índice de circulación modificado:

$$
\frac{C}{C_{1}}=\frac{\frac{\bar{m}}{\bar{l}}\left(1+\frac{\bar{l}}{\bar{n}}\right)\left(1+\frac{\bar{n}}{\bar{N}}\right)}{\frac{\bar{m}_{i}}{\bar{l}_{i}}\left(1+\frac{\bar{l}_{i}}{\bar{n}_{i}}\right)\left(1+\frac{\bar{n}_{i}}{\bar{N}_{i}}\right)}
$$

Cuyaex pre sión ofre ce la me di da com parada de la frecuencia de préstamos por lec tor en dos mo men tos dis tin tos e in corpora a esas medidas los valores de la comunicación y la captación, indicando también el rendimiento por bibliotecario en cada una de las variables utilizadas.

Entonces, el índice de comportamiento (IC) constituye una fun ción de las va riables pre sen tes en la ac ti vi dad bi blio te caria, que ex pre san efi cien cia, me dia do por los índices que acaban de exponerse:

$$
f \frac{\frac{\bar{m}}{\bar{m}_{i}}}{\frac{\bar{f}_{i}}{\bar{f}_{i}}}, \frac{\frac{\bar{m}}{l}\left(1+\frac{\bar{l}}{\bar{n}}\right)\left(1+\frac{\bar{n}}{\bar{N}}\right)}{\frac{\bar{m}_{i}}{\bar{l}_{i}}\left(1+\frac{\bar{l}_{i}}{\bar{n}_{i}}\right)\left(1+\frac{\bar{n}_{i}}{\bar{N}_{i}}\right)}
$$

Como ya fue señalado, IC es un número integral de com porta mien to conjun to. Se propone promediar el comportamiento de índices parciales, como medida integral, para lo que se utiliza la media geométrica $(M g)$, lo que cons ti tu ye una aplicación original de esta medida de tendencia central a la actividad biblioteca ria. Es opor tu no re cor dar que la me dia geo métricaseempleacuan do se nece sita promediar razones o tasas de crecimiento. $M g$ se de fi ne como el va lor que re sul ta de estimar la raíz enésima del producto de $n$ observaciones: 5

$$
M g=n \sqrt{x_{1} \cdot x_{2} \cdots x_{n}}
$$

luego, en el índice de comportamiento:

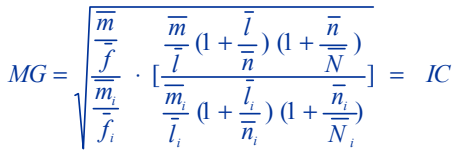

$$
\begin{aligned}
& \overline{\text { recursos }} \overline{\text { efectividad }}
\end{aligned}
$$


La formulación del modelo pudiera ser objeto de cier to grado desimplificación, pero esto con du ci ría a la pér di da de in formación explícita sobre los índices de rota ción y circu la ción, rom pien do asíla posibilidad de analizar el comportamiento de conceptos clave para llegar a conclusiones so bre el com por ta mien to de la eficiencia.

Las biblio te cas públicas cu ba nas se diseñan tomando como base ciertas recomendaciones proporcionales para este tipo de ins ti tu cio nes: 2 vo lú me nes en colec ción por lec tor po ten cial; 1 biblio tecario cada 2000 lec to res po ten cia les. La situación de una de estas instituciones en dos años consecutivos es la siguiente:

\begin{tabular}{|l|l|l|}
\hline & \multicolumn{1}{|c|}{ Año A } & \multicolumn{1}{|c|}{ Año B } \\
\hline Lectores potenciales & 4048 & 4976 \\
\hline Colecciones & 8200 vol. & 9880 vol. \\
\hline Préstamos & 24602 & 24700 \\
\hline $\begin{array}{l}\text { Lectores reales } \\
\text { inscritos }\end{array}$ & 1398 & 1512 \\
\hline $\begin{array}{l}\text { Lectores que } \\
\text { utilizan el fondo }\end{array}$ & 984 & 1030 \\
\hline Bibliotecarios & 2 & 2 \\
\hline
\end{tabular}

Estos índices, expresados en rendimiento por bibliotecario, son:

\begin{tabular}{|l|l|l||}
\hline & \multicolumn{1}{|c|}{ Año A } & \multicolumn{1}{|c|}{ Año B } \\
\hline Lectores potenciales & 2024 & 2488 \\
\hline Colecciones & 4100 vol. & 4940 vol. \\
\hline Préstamos & 12301 & 12350 \\
\hline $\begin{array}{l}\text { Lectores reales } \\
\text { inscritos }\end{array}$ & 699 & 756 \\
\hline $\begin{array}{l}\text { Lectores que } \\
\text { utilizan el fondo }\end{array}$ & 492 & 515 \\
\hline
\end{tabular}

Sustituyendo valores en el modelo de comportamiento, tomando el año A como base (denominador), tenemos:

$$
I C=\sqrt{\frac{\frac{12,350}{4,940} \cdot \frac{12,350}{515}\left(1+\frac{515}{756}\right)\left(1+\frac{756}{2,488}\right)}{\frac{12,301}{4,100} \cdot \frac{12,301}{492}\left(1+\frac{492}{699}\right)\left(1+\frac{699}{2,024}\right)}}
$$

$$
\begin{gathered}
I C=\sqrt{\frac{2.5}{3} \cdot \frac{24(1.68)(1.30)}{25(1.70)(1.35)}} \\
I C=\sqrt{0.83 \times 0.91}=0.87
\end{gathered}
$$

La eficiencia de la biblioteca disminuyó globalmente en 13 puntos de un año a otro. Mien tras que los ín di ces de cir cu lación, comunicación y captación experimentan disminuciones mínimas, la más sen si ble se pro du ce en los va lo res del índice de rotación. Esto puede tener sus causas en dos razones:

a) Las adquisiciones del periodo no se ajustan satisfactoriamente a las demandas de los lectores (véase al respecto el artículo de Stoljarov). ${ }^{6}$

b) El cre ci mien to al can za do por la colección sobrepasa las posibilidades de la fuerza de trabajo para atenderlas eficientemente(nótese que en un año las colecciones crecieron en más del $20 \%)$.

Consecuentemente, la biblioteca debe orientar su análisis hacia estas dos posibilidades para determinar cuál incidió real men te en la dis mi nu ción de la eficiencia.

La creación del modelo de comportamiento de las bibliotecas públicas cubanas tiene sus antecedentes en el coeficiente de intensidad de la actividad bibliotecaria de Medinski y Rubakin ${ }^{7}$ y en una de las medidas de efectividad de las bibliotecas empleadas por Rzasa y Baker. ${ }^{8}$

El coe fi cien te de in ten si dad de Me dinskiy Rubakin se expresa de la forma siguiente:

$$
C I=C \cdot R
$$

donde

$C=$ índicedecirculación $=m / N$ (préstamo por lector potencial), y

$R=$ índice de rotación $=m / f$ (préstamos por volumen)

Aquí $C$ presenta como limitante, de acuerdo con nuestros intereses, el referir el promedio de préstamos a los lectores po ten ciales, lo que no per mi te co no cer la fre cuen cia real de lec tu ra por usua rio. En el modelo ahora propuesto, esta dificul-

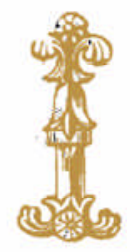

La eficiencia de la biblioteca disminuyó globalmente en 13 puntos de un año a otro. Mientras que los índices de circulación, comunicación y captación experimentan disminuciones mínimas, la más sensible se produce en los valores del índice de rotación

6 Véase Stoljarov, Ju. N. “Amplitud óptima de los fondos de bibliotecas públicas”. Boletín de la Unesco para las bibliotecas (París) 27(1): 23-29; enero-febrero, 1973.

7 Véase Frumin, I.M. Organizatsia raboti sovetskoi biblioteki. — Moskva : Kniga, 1969. — p. 162.

8 Véase Philip V. Rzasa y Norman R. Baker. "Measures of Effectiveness for a University Library”. Journal of the American Society of Information Sciences (Washington) 23(4):248-253; July-August, 1977. 


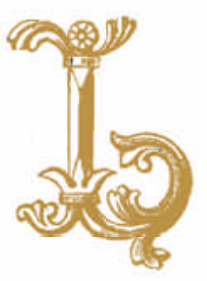

El modelo de comportamiento permite expresar en un índice integral el valor teórico (calculado) de la relación que existe entre la eficiencia real de las bibliotecas y los valores de base que se toman como referencia en tanto se encuentran reflejados en él los esfuerzos fundamentales de esas instituciones

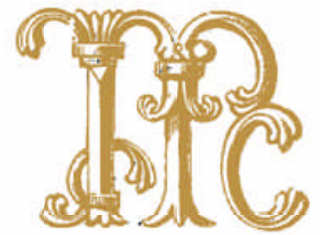

tad se sal va al uti li zar el con cep to de lectores que utilizan el fondo $(l)$.

Entre las medidas de efectividad de las bibliotecas de Rzasa y Baker estos autores consideran igualmente el índice de circulación, pero re fie ren el pro me dio de préstamos a los lectores reales inscritos en las bi blio te cas (ha gan uso del fon do o no) y agre gan al va lor delín di ce de cir culación el va lor delín di ce de cap ta ción de estas instituciones.

$$
C=\frac{m}{n}\left(1+\frac{n}{N}\right)
$$

donde

$C=$ índice de circulación $=m / n$ (préstamo por lector real inscrito en la biblioteca), y

$n / N=$ índice de captación (lectores reales inscritos en las bibliotecas/lectores potenciales)

Se presenta nue va men te un pro me dio de prés ta mos por lec tor que no re fle ja la frecuencia real de lectura; sin embargo, se añadeacertadamente al valor de la circulación el de la captación, teniendo en cuenta que ésta es un requisitoindispensable para lograr los préstamos.

El modelo de comportamiento de las bibliotecas públicas cubanas incorpora, entonces, como elemento novedoso, el índice de comunicación:

$l / n$ (lectores que utilizan el fondo/lectoresrealesins critos en las bibliotecas)

Este índice es útil porque representa un comportamiento típico de los lectores que asis ten a las bi blio te cas pú bli cas cubanas, los cuales se dividen en dos grupos: aque llos que uti lizan las co lec ciones (l) y los que sólo se sirven de la instalación como local de estudio o de trabajo $(n-l)$, porque acuden a ella con sus propios materiales de consulta. El índice de comunicación expresa en alguna medida el pa pel orien ta dor de las bi blio te cas, por lo que constituye también un elemento que propicia los préstamos y conviene añadir su valor al del índice de circulación.
Nó te se que en nin gu no de los dos an te cedentes citados se comparan los valores reales de un momento determinado, con valores de base que permitan analizar el devenir de la actividad bibliotecaria, lo que síse con si de ra en el mo de lo de comportamiento.

En conclusión, el modelo de comportamiento permite expresar en un índiceintegral el valor teórico (calculado) de la re lación que exis te en tre la efi cien cia real de las bibliotecas y los valores de base que se toman como referencia, en tanto se encuentran refle ja dos en él los es fuerzos fundamentales de esas instituciones —dados por los recursos de colección y el rendimiento del personal-, para lograrsuefec ti vidad, ex presada porelíndice de circulación, el grado de comunicación social alcanzado, el poder de captación y el alcance social del fondo existente.

Elíndice de comporta mien to (IC) que se logra con el modelo diseñado expresa, portan to, en sín te sis, la co rres pon den cia existente entre la eficiencia real y la que se toma como base para la com pa ra ción.

Cuan do el va lor de $I C=1$, esa correspondencia es total; cuando $I C<1$, no existe correspondencia entre la eficiencia real (estudiada) y la que se toma como base, por de fec to de la pri me ra res pec to a la segunda; cuando $I C>1$ esa correspondencia tampoco existe, en este caso, por exce so de la primerares pec to a la se gunda.

Un elemento fundamental obtenido duran te la in ves ti ga ción y to ma do en con sideración al aplicar el modelocorresponde a la redefinición del concepto de lectores potenciales. Se considera como tales a:

Los habitan tes que re si den en el ra dio de acción de $2 \mathrm{~km}$. como máximo, alrededor de cada biblioteca pública, para los que se cuenta con fondo suficiente, calcu la do se gún el percápi ta de volú me nes por lec tor po ten cial adop ta do en los planes de desarrollo de esas instituciones. 